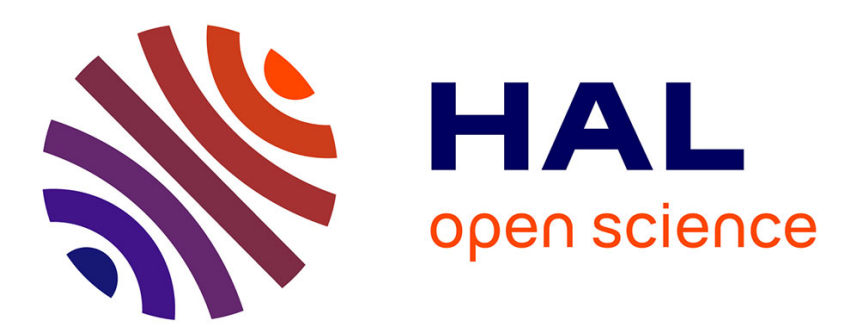

\title{
Theoretical studies of vibrational relaxation of iodine in low density liquid xenon
}

\author{
J.K. Brown, D.J. Russell, D.E. Smith, C.B. Harris
}

\section{To cite this version:}

J.K. Brown, D.J. Russell, D.E. Smith, C.B. Harris. Theoretical studies of vibrational relaxation of iodine in low density liquid xenon. Revue de Physique Appliquée, 1987, 22 (12), pp.1787-1792. 10.1051/rphysap:0198700220120178700 . jpa-00245740

\section{HAL Id: jpa-00245740 https://hal.science/jpa-00245740}

Submitted on 1 Jan 1987

HAL is a multi-disciplinary open access archive for the deposit and dissemination of scientific research documents, whether they are published or not. The documents may come from teaching and research institutions in France or abroad, or from public or private research centers.
L'archive ouverte pluridisciplinaire HAL, est destinée au dépôt et à la diffusion de documents scientifiques de niveau recherche, publiés ou non, émanant des établissements d'enseignement et de recherche français ou étrangers, des laboratoires publics ou privés. 


\title{
Theoretical studies of vibrational relaxation of iodine in low density liquid xenon
}

\author{
J. K. Brown, D. J. Russell, D. E. Smith and C. B. Harris \\ Department of Chemistry, University of California, Berkeley, California 94720, U.S.A. \\ Materials and Chemical Sciences Division, Lawrence Berkeley Laboratory, Berkeley, California 94720 , \\ U.S.A.
}

(Reçu le 12 juin 1987, accepté le 24 août 1987)

\begin{abstract}
Résumé. - Des résultats préliminaires de calculs utilisant le modèle de Langevin généralisé et de collisions binaires isolées (IBC) pour la relaxation vibrationnelle de l'iode dans le xénon liquide à faible densité sont présentés. Des simulations de Langevin généralisées simples, utilisant le xénon à l'équilibre comme modèle de solvant, ne reproduisent pas les données de la dynamique moléculaire sur la relaxation vibrationnelle. Ceci est expliqué, au moins en partie, en remarquant que le mouvement vibrationnel de l'iode perturbe localement le solvant et que cette perturbation se traduit par une dissipation d'énergie. Des modèles simples de collisions isolées binaires reproduisent qualitativement le comportement de relaxation. Cependant, le taux de collision déterminé en ajustant les données de collisions binaires isolées aux données de la dynamique moléculaire semble anormalement élevé, ce qui peut indiquer l'importance des collisions corrélées et des interactions à plusieurs corps qui sont négligées dans le modèle IBC.
\end{abstract}

\begin{abstract}
Preliminary results of generalized Langevin and isolated binary collision (IBC) calculations for vibrational relaxation of iodine in low density liquid xenon are presented. Simple generalized Langevin simulations, using equilibrium xenon as a model solvent, fail to reproduce molecular dynamics vibrational relaxation data. This is explained, at least in part, by noting that the iodine vibrational motion perturbs the local solvent, with this perturbation resulting in dissipation of energy. Simple isolated binary collision models qualitatively reproduce molecular dynamics relaxation behaviour. However, the collision rate determined by fitting isolated binary collision data to molecular dynamics data seems unreasonably high, which may indicate the importance of correlated collisions and many body interactions which are ignored in the IBC model.
\end{abstract}

\section{Introduction.}

The study of simple chemical reactions in liquids, and in particular vibrational relaxation, has progressed rapidly in the past decade. Most of the advances are due to improvements in the picosecond and subpicosecond laser technology required to measure rapid processes, and to the availability of computers capable of performing moderate size model simulations. In this paper, we will discuss preliminary results of the merger of these tools in our recent research efforts on the photodissociation/recombination reaction of iodine in various solvents. The goal of this work is to determine the properties of the reactants and the solvent that must be included in a qualitative description of any simple condensed phase reaction, and to develop simple predictive and semiquantitative models from this information.

The photodissociation/recombination of iodine has been studied extensively since Franck and Rabi- nowitch predicted the cage effect in 1934 [1]. Until the mid 70's, most of the experimental and theoretical work on iodine in various liquids was directed at understanding the short time dynamics of the dissociation process through indirect probes such as geminate recombination probabilities [2-9]. The first direct dynamic experiments of iodine in solution on a picosecond timescale were performed by Chuang et al. [10] ; since these initial experiments, many groups of workers have contributed to the understanding of various details of this model chemical reaction [11-42] — including photoexcitation, predissociation, excited state curve crossing, geminate recombination and vibrational relaxation. Our current work in this field focuses on ground electronic state vibrational relaxation of iodine in liquid xenon. In this paper, we will outline preliminary results of our attempts to model the vibrational relaxation process as observed in molecular dynamic simulations of $I_{2}$ in liquid xenon. Although some experi- 
mental data is currently available for this system [12], molecular dynamics is the basis of our initial comparisons since detailed information regarding potentials and various force correlation functions are readily obtained from such simulations.

Theories of vibrational relaxation of iodine in simple model liquids have typically come from two extreme points of view. The first class of theories are based on the hydrodynamic idea that collective motions of the solvent are dominant in the relaxation of an excited oscillator [35, 37]. These theories ignore explicit solvent-reactant interactions, and are expected to best apply to oscillators with relatively low vibrational frequencies. The second class is that of isolated binary collision models, where it is assumed that the infrequent hard collisions of the solvent with the relaxing oscillator dominate the vibrational relaxation process $[17,41]$. These models neglect correlated and higher order collisions, and are typically expected to be most appropriate for high vibrational frequencies. Models based on both of these types of theories attempt to drastically reduce the number of degrees of freedom necessary to simulate the vibrational relaxation process, and hopefully replace them with experimentally accessible parameters.

Results for two types of models will be discussed in this paper. The first are hydrodynamic type simulations based on Langevin equations and generalized Langevin equations. The second type of simulations are classical one-dimensional trajectories with one solvent atom and one vibrationally excited oscillator. These models are compared with molecular dynamic simulations of iodine in low density liquid xenon $(1.8 \mathrm{~g} / \mathrm{cc})$. The details of the molecular dynamic simulations are discussed elsewhere [42].

\section{Langevin models.}

Langevin and generalized Langevin theories for chemical reactions in solution are based on replacing detailed solvent interactions with appropriately modeled random and dissipative forces. In their simplest form, the motion of the particle of interest is described by the Langevin equation.

$$
m \dot{v}(t)=-\gamma_{0} v(t)+R(t)
$$

$\gamma_{0}$ is the solvent coefficient of friction and $R(t)$ is a Gaussian distributed random driving force with the following properties :

$$
\begin{aligned}
\langle R(0) R(t)\rangle & =2 k T \gamma_{0} \delta(t) \\
\langle R(t)\rangle & =0 .
\end{aligned}
$$

Equation (2) is the fluctuation dissipation theorem and ensures that the correct equilibrium temperature is reached. Detailed solvent interactions are thus replaced by a simple stochastic equation, the solvent being characterized only by its coefficient of friction and the temperature. Implicit in this simple approach is the assumption that correlations in the "random » force are short relative to the timescale for significant variation in the particle velocity. This assumption is appropriate for very large particles but fails completely for small chemical systems where single collisions significantly change particle velocities. Thus to adequately model simple chemical reactions in solution it is necessary to turn to generalized Langevin models which avoid such unrealistic assumptions.

The generalized Langevin equation of motion for a particle in one dimension can be written as [43] :

$$
m \dot{v}(t)=-\int \gamma(t-\tau) v(\tau) \mathrm{d} \tau+R(t)
$$

with

$$
\begin{aligned}
\langle R(0) R(t)\rangle & =k t \gamma(t) \\
\langle R(t)\rangle & =0 .
\end{aligned}
$$

The random force, $R(t)$, now has a finite correlation time and the simple dissipative term in equation (1) has been replaced by a friction integral. Equation (3) reduces to equation (1) if $\gamma(t)=2 \gamma_{0} \delta(t)$. The solvent is now characterized by a time (or frequency) dependent friction as opposed to simply the zerofrequency friction in the Langevin case. The primary difficulties in using equation (3) to simulate the effects of the solvent on a chemical reaction are in determining the correct form for $\gamma(t)$ and in generating random forces with correlations proportional to $\gamma(t)$.

TOTAL FORCE RUTOCORRELATION FUNCTION

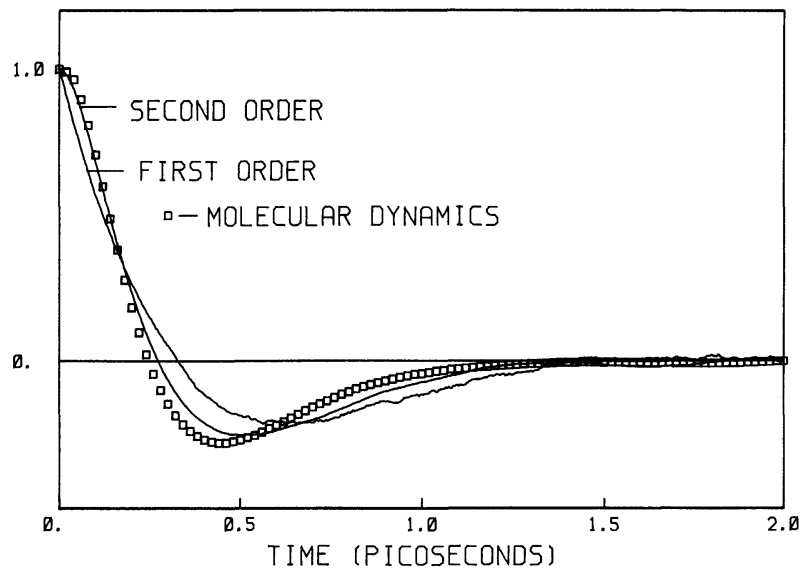

Fig. 1. - Normalized total force autocorrelation functions generated using first and second order approximations for $\gamma(t)$. (First order : $a=5.0 \mathrm{ps}^{-1}$; second order : $a=10.05 \mathrm{ps}^{-1}, \quad b=10.30 \mathrm{ps}^{-1}$.) Comparison is made with the equilibrium xenon facf generated from a molecular dynamics simulation. 
As a first approximation, we have chosen to model $\gamma(t)$ after the equilibrium Xe-Xe force autocorrelation function (facf). This seems to be a reasonable first choice since $I_{2}$ is similar in size to xenon and the $I_{2}-\mathrm{Xe}$ potential is similar to the Xe-Xe potential. A molecular dynamics simulation was used to generate the xenon facf, which is shown in figure 1. A problem immediately arises in that $\gamma(t)$ is proportional to the random facf while the molecular dynamics correlation function is a total facf; however, the early time (high frequency) portion of the total facf is dominated by the random forces, and can therefore be used to fit to $\gamma(t)$ [43]. Correlated random numbers are generated by a simple technique in which each random number is correlated with the previous one or two random numbers :

$$
\begin{array}{ll}
x_{n}=\alpha x_{n-1}+\varepsilon_{n} & \text { first order } \\
x_{n}=\alpha x_{n-1}+\beta x_{n-2}+\varepsilon_{n} & \text { second order }
\end{array}
$$

where $\varepsilon_{n}$ is a Gaussian distributed random number with $\left\langle\varepsilon_{n}\right\rangle=0$. These lead to :

$$
\begin{array}{ll}
\gamma(t) \propto \mathrm{e}^{-a t} & \text { first order } \\
\gamma(t) \propto \frac{\mathrm{e}^{-a t}}{a}-\frac{\mathrm{e}^{-b t}}{b} & \text { second order }
\end{array}
$$

where the coefficients $a$ and $b$ are functions of $\alpha$ and $\beta$. Given the form of $\gamma(t)$, calculation of particle trajectories proceeds by numerically integrating the friction integral in equation (3) and adding this to the random force. Calculated total facf's for a single particle, using both first and second order approximations for $\gamma(t)$ fit to the early time portion of the $\mathrm{Xe}-\mathrm{Xe}$ facf, are shown in figure 1. Comparison shows that the first order approximation fails to closely model the early time feature, as expected given the non-zero slope of the first order approximation for $\gamma(t=0)$. (This non-zero slope has the important consequence of increasing the amplitude of high frequency components in the first order power spectrum, as will be discussed below.) The second order approximation for $\gamma(t)$ includes the proper slope at $t=0$, and therefore fits the Xe-Xe facf much more closely at early time, and in fact the fit is reasonably good even at long time.

Equation (3) is now used with the first and second order approximations for $\gamma(t)$ to simulate vibrational relaxation of iodine. The equation of motion for each iodine atom is given by equation (3) plus an additional force term due to the I-I potential. The random force is applied independently to each iodine atom. The iodine vibrational frequency varies from about $150 \mathrm{~cm}^{-1}$ near the top of the well to $214 \mathrm{~cm}^{-1}$ at the bottom of the well, gradually increasing as the molecule relaxes. In order to qualitatively predict the efficiency of energy transfer to our model solvent it is helpful to look at the

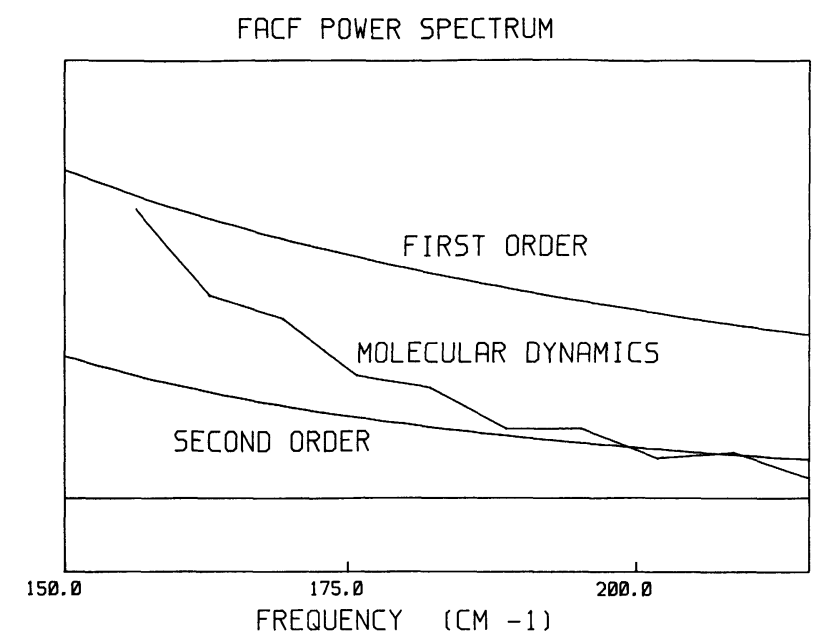

Fig. 2. - Power spectra for the equilibrium xenon total force autocorrelation function and the first and second order approximations for $\gamma(t)$ in the frequency region of iodine vibrational motion.

frequency dependence of $\gamma(t)$. The power spectra of the xenon facf and the first and second order approximations for $\gamma(t)$ are shown in figure 2. The relaxation rate of iodine at a given vibrational frequency should be approximately proportional to the magnitude of that frequency component in the facf power spectrum. Thus, the first order simulation should show more rapid relaxation than the second order simulation, with the second order simulation more closely matching the « real » relaxation rate from molecular dynamics. In fact (Fig. 3), the first order simulation does show significantly faster relaxation than either the second order or molecular dynamics result, consistent with our prediction. However, there is a significant difference between the second order relaxation and the molecular

I2 VIBRATIONAL RELAXATION

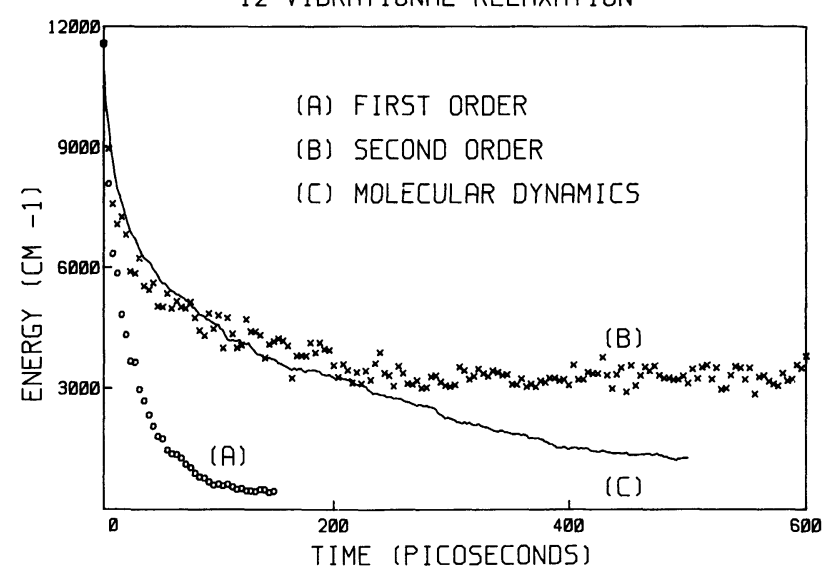

Fig. 3. - Average iodine vibrational energy versus time calculated for both first and second order model solvents and compared to molecular dynamics. 
dynamics result, with the Langevin relaxation rate appreciably slower than the molecular dynamics rate in the bottom third of the well. This notable difference cannot be explained by differences in the xenon and second order power spectra.

To explain this difference in relaxation rates it is necessary to go back to the original approximation of modeling the I-Xe facf by the equilibrium xenon facf. Figure 4 shows the equilibrium xenon power spectrum along with an iodine-xenon facf power spectrum. The iodine-xenon facf power spectrum shown in figure 4 is a correlation function of the force exerted by the xenon solvent on the iodine vibrational coordinate as determined from molecular dynamics simulations. The power spectra show there

FACF POWER SPECTRA

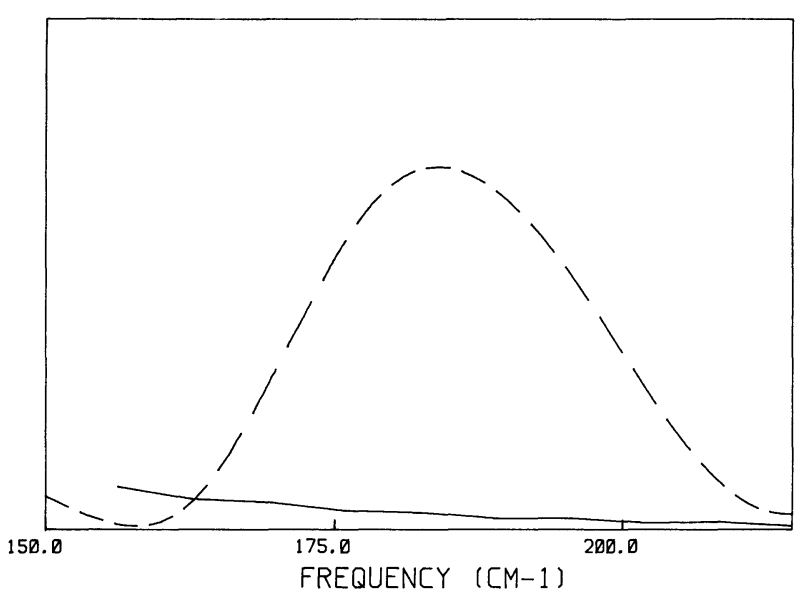

Fig. 4. - Comparison of xenon-xenon and iodine-xenon facf power spectra in the frequency region of iodine vibrational motion. (Dashed line: iodine-xenon power spectrum, $\sim 4000 \mathrm{~cm}^{-1}$ vibrational energy; solid line : xenon-xenon power spectrum $(\times 10)$.)

are large frequency components near the iodine vibrational frequency, with the magnitude of these components varying with vibrational energy. However, the large magnitude of these components alone does not ensure coupling of the iodine vibrational motion to the xenon solvent. For example, the xenon atoms could be essentially at rest over one iodine vibrational period ; and therefore, the component of the facf power spectrum at the iodine vibrational frequency would simply be indicative of the approach and recession of the iodine atoms to the static first xenon solvation shell over the period of one vibration. For this type of motion, the force on the iodine vibrational coordinate due to the iodine potential is in phase with the force on the iodine vibrational coordinate due to the xenon solvent, and does not lead to the dissipation of vibrational energy. However, an approximate phase shift was calculated from the crosscorrelation func- tion of the solvent forces and the internal oscillator forces from molecular dynamics simulations, and phase shifts on the order of $20^{\circ}$ were typical for several solvent densities and iodine vibrational energies. Therefore, we conclude that the iodine vibrational motion does perturb the local solvent, especially at higher vibrational energies, and that this perturbation does lead to the dissipation of energy. This also indicates a weakness of using only the equilibrium solvent force autocorrelation function when constructing models of vibrational relaxation based on the generalized Langevin equation, especially if the characteristic vibrational frequency is significantly greater than the Debye frequency of the solvent. As an extension of this work it would be possible to construct random forces which more closely model the I-Xe facf power spectra [44, 45]. Although this would be instructive as a test of the applicability of generalized Langevin models to vibrational relaxation, any approach requiring detailed knowledge of time and phase dependent facfs currently would have little applicability as a predictive model for vibrational relaxation.

\section{Isolated binary collision model.}

Perhaps the simplest model for describing vibrational relaxation in a liquid is the isolated binary collision model (IBC) [46]. This model was compared to the molecular dynamics simulation results because of IBC's simplicity and widespread use in modeling vibrational relaxation, including the $\mathrm{I}_{2}$ experiments [17]. The IBC model assumes that the relaxation rate is the product of the probability of relaxation per collision and the collision rate. Applying this model in a liquid makes the additional assumption that, although the oscillator is interacting with many solvent molecules at once, only the relatively rare hard collisions are important. Assuming the relaxation probabilities are known from the gas phase or are otherwise calculated, the relaxation rate in a liquid can be determined by assuming an appropriate collision rate for the hard collisions. This implies that at different solvent densities the curve of energy $v s$. time should have the same functional form. Only the time axis must be changed to scale the collision rates at the different densities. The model neglects correlated collisions and in its simplest form many body interactions [47, 48]. A simple criterion for measuring the importance of correlated collisions is that $\omega \tau_{\mathrm{c}} \gg 1$, where $\omega$ is the vibrational frequency and $\tau_{c}$ is the time between hard collisions [49]. Although $\mathrm{I}_{2}$ in liquid xenon does not meet the above criteria, and the IBC model should therefore fail to describe the $I_{2}$ relaxation, it is still instructive to compare an IBC calculation to the molecular dynamics results.

The IBC simulations discussed here are very 
similar to earlier calculations by Nesbitt and Hynes [41]. The main differences are that here the $\mathrm{I}_{2}$ molecule is bound by an RKR potential and the I$\mathrm{Xe}$ force is not exponentially repulsive. The $\mathrm{I}-\mathrm{Xe}$ potential used in this paper is a WCA decomposition of a Lennard-Jones potential [50]. These different potentials were used in order to test the sensitivity of the calculation to the potential. The results of the two calculations are similar both qualitatively and in the time scale for relaxation. This suggests that the calculation is relatively insensitive to small changes in the potential, which is supported further by similar calculations (work in progress). It must be pointed out that the WCA decomposition was originally intended to explain liquid structure for reduced densities $\left(\rho^{*}\right)$ greater than 0.6. The IBC calculations will be compared to liquid $\mathrm{Xe}$ molecular dynamics calculations at slightly lower $\rho *$ under the assumption that the attractive part of the potential thrown away in the WCA decomposition will not contribute significantly to relaxation.

Figure 5 shows a comparison of molecular dynamics and IBC results where a collision rate of approximately 4.5 collisions per ps. is assumed. The curves are the same qualitatively. In both calculations the rate of energy loss is higher in the top of the $I_{2}$ well, where the vibrational frequency is lower and the vibrational amplitude is higher, and slows as the $I_{2}$ relaxes. Although the curves are qualitatively the same, the collision rate that must be assumed for the IBC calculation to fit the molecular dynamics results is found to be too high. A collision rate can be extracted from the molecular dynamics simulation by assuming that every solvent atom that comes within a certain radius of the $I_{2}$ molecule is a collision. Collision frequencies calculated in this way with physically reasonable radii are at least a factor of two lower than the collision frequency needed to fit the IBC results to molecular dynamics. Also it must be remembered that the IBC calculation is for a collinear trajectory, which is most efficient for energy exchange, while the molecular dynamics collision rates are determined from a three-dimensional simulation. Therefore, a geometric factor between zero and one should be included in the IBC results, which will further increase the discrepancy between the IBC and molecular dynamics results. The high collision rate needed for the IBC calcula-

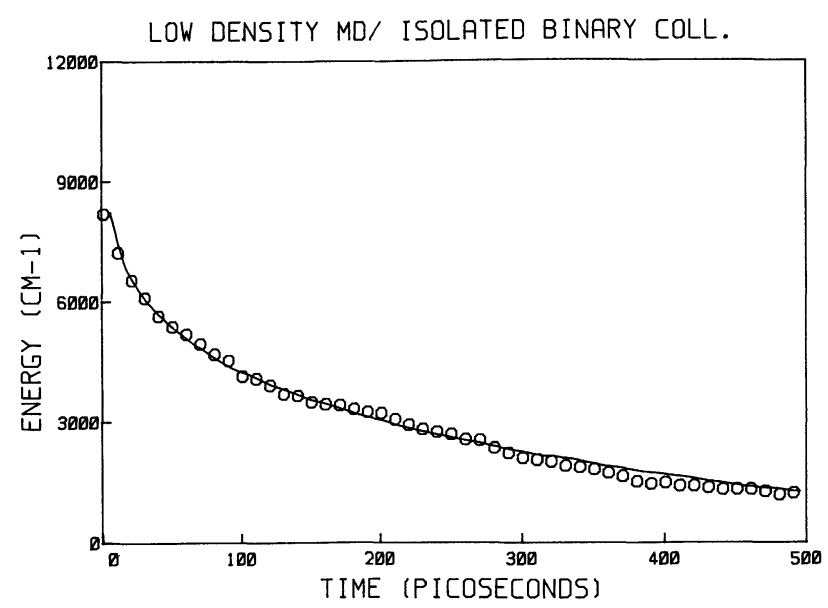

Fig. 5. - Average iodine vibrational energy versus time calculated for molecular dynamics (circles) and isolated binary collision model (solid line).

tion may indicate the extent to which correlated collisions and many body interactions, ignored in the IBC model, contribute to vibrational relaxation.

\section{Conclusions.}

We have presented here preliminary results of generalized Langevin and isolated binary collision calculations for vibrational relaxation of iodine in liquid xenon. Simple generalized Langevin simulations, using equilibrium xenon as a model solvent, fail to reproduce molecular dynamics vibrational relaxation data. This is explained, at least in part, by noting that the iodine vibrational motion perturbs the local solvent, with this perturbation resulting in dissipation of energy. Simple isolated binary collision models qualitatively reproduce molecular dynamics relaxation behaviour. However, the collision rate determined by fitting isolated binary collision data to molecular dynamics data seems unreasonably high, which may indicate the importance of correlated collisions and many body interactions which are ignored in the IBC model.

\section{Acknowledgments.}

This work was supported by the National Science Foundation. Molecular dynamics simulations were performed at the San Diego Supercomputer Center.

\section{References}

[1] Franck, J. and Rabinowitch, E., Trans. Faraday Soc. 30 (1934) 120.

[2] Zimmermann, J. and Noyes, R. M., J. Chem. Phys. 18 (1950) 658.

[3] Lampe, F. W. and Noyes, R. M., J. Am. Chem. Soc. 76 (1954) 2140.
[4] Booth, D. and Noyes, R. M., J. Am. Chem. Soc. 82 (1960) 1868.

[5] Meadows, L. F. and Noyes, R. M., J. Am. Chem. Soc. 82 (1960) 1872.

[6] Noyes, R. M., Z. Electrochem. 64 (1960) 153.

[7] Noyes, R. M., Prog. React. Kinet. 1 (1961) 129. 
[8] Bunker, D. L. and Jacobson, B. S., J. Am. Chem. Soc. 94 (1972) 1843.

[9] Murrell, J. N., Stace, A. J. and Dammell, R., J. Chem. Soc. Faraday Trans. 2. 74 (1978) 1532.

[10] Chuang, T. J., Hoffman, G. W. and Eisenthal, K. B., Chem. Phys. Lett. 25 (1974) 201.

[11] Harris, A. L., Berg, M. and Harris, C. B., J Chem. Phys. 84 (1986) 788.

[12] Paige, M. E., Russell, D. J. and Harris, C. B., J. Chem. Phys. 85 (1986) 3699.

[13] Smith, D. E. and Harris, C. B., J. Chem. Phys. (in press).

[14] Berg, M., Harris, A. L. and Harris. C. B., Phys. Rev. Lett. 54 (1985) 951.

[15] Berg, M., Harris, A. L., Brown, J. K. and HARrIS, C. B., in Ultrafast Phenomena IV, Eds D. H. Auston and K. B. Eisenthal, Springer Series in Chemical Physics (Springer, New York) 1984, Vol. 38, p. 300.

[16] Harris, C. B., Brown, J. K., Paige, M. E., Smith, D. E. and RUSSEL, D. J., in Ultrafast Phenomena $V$, Eds G. R. Fleming and A. E. Siegman, Springer Series in Chemical Physics (Springer, New York) 1986, Vol. 46, p. 326.

[17] Abul-HaJ, N. A. and Kelley, D. F., J. Chem. Phys. 84 (1986) 1335.

[18] Kelley, D. F., Abul-HaJ, N. A. and Jang, D.-J., J. Chem. Phys. 80 (1984) 4105

[19] Kelley, D. F. and Abul-HaJ, N. A., in Ultrafast Phenomena $I V$, Eds D. H. Auston and K. B. Eisenthal, Springer Series in Chemical Physics (Springer, New York) 1984, Vol. 38, p. 292.

[20] Kelley, D. F. and Rentzepis, P. M., Chem. Phys. Lett. 85 (1982) 85.

[21] Abul-Haj, N. A. and Kelley, D. F., Proc. Soc. Photo-Opt. Instrum. Eng. 533 (1985) 20.

[22] Bado, P., Dupuy, C., Magde, D., Wilson, K. R. and Malley, M. M., J. Chem. Phys. 80 (1984) 5331.

[23] Bado, P. and Wilson, K. R., J: Phys. Chem. 88 (1984) 655.

[24] Bado, P., Berens, P. H., Bergsma, J. P., Wilson, S. B., Wilson, K. R. and Heller, E. J., in Picosecond Phenomena III, Eds K. B. Eisenthal, R. M. Hochstrasser, W. Kaiser and A. Laubereau, Springer Series in Chemical Physics (Springer, New York) 1982, Vol. 23, p. 260.

[25] Bado, P., Dupuy, C. G., Bergsma, J. P. and WILSON, K. R., in Ultrafast Phenomena IV, Eds D. H. Auston and K. B. Eisenthal, Springer
Series in Chemical Physics (Springer, New York) 1984, Vol. 38, p. 296.

[26] Langhoff, C. A., Moore, B. and de Meuse,M., J. Am. Chem. Soc. 104 (1982) 3576.

[27] Langhoff, C. A., Moore, B., and de Meuse, M., J. Chem. Phys. 78 (1983) 1191.

[28] Stace, A. J. and Murrell, J. N., Mol. Phys. 33 (1977) 1 .

[29] Evans, G. T. and Fixman, M., J. Phys. Chem. 80 (1976) 1544.

[30] Martire, B. and Gilbert, R. G., Chem. Phys. 56 (1981) 241.

[31] Hynes, J. T., Kapral, R. and Torrie, G. M., J. Chem. Phys. 72 (1980) 177.

[32] Schell, M. and Kapral, R., Chem. Phys. Lett. 81 (1981) 83.

[33] Shin, K. J. and Kapral, R., J. Chem. Phys. 69 (1978) 6385.

[34] Adelman, S. A., Adv. Chem. Phys. 53 (1983) 61.

[35] Brooks III, C. L., Balk, M. W. and Adelman, S. A., J. Chem. Phys. 79 (1983) 784.

[36] Balk, M. W., Brooks III, C. L. and Adelman, S. A., J. Chem. Phys. 79 (1983) 804.

[37] Brooks III, C. L. and Adelman, S. A., J. Chem. Phys. 80 (1984) 5598.

[38] Dawes, J. M. and Sceats, M. G., Chem. Phys. 96 (1985) 315.

[39] Lipkus, A. H., Buff, F. P. and Sceats, M. G., J. Chem. Phys. 79 (1983) 4830.

[40] Ali, D. P. and Miller, W. H., J. Chem. Phys. 78 (1983) 6640 ; Chem. Phys. Lett. 105 (1984) 501.

[41] Nesbitt, D. J. and Hynes, J. T., J. Chem. Phys. 77 (1982) 2130.

[42] Brown, J. K., Harris, C. B., and Tully, J. C. (in preparation).

[43] Kubo, R., Rep. Prog. Theor. Phys. 29 (1966) 255. See also,

Mori, H., Progr. Theor. Phys. 33 (1965) 423.

Zwanzig, R., Ann. Rev. Phys. Chem. 16 (1965) 67.

[44] Ciccotti, G. and Ryckaert, J.-P., Mol. Phys. 40 (1980) 141.

[45] Ciccotti, G., Ferrario, M., and Ryckaert, J.-P., Mol. Phys. 46 (1982) 875.

[46] Maidgosky, W. M. and Litovitz, T. A., J. Chem. Phys. 34 (1961) 489.

[47] Davis, P. K. and Oppenheim, I. J., J. Chem. Phys. 57 (1972) 505.

[48] Chesnoy, J. and Gale, G. M., Ann. Phys. 6 (1984) 893.

[49] Zwanzig, R. J., J. Chem. Phys. 34 (1962) 1931.

[50] Andersen, H. C., Chandler, D. and Weeks, J. D., Adv. Chem. Phys. 34 (1976) 105. 